\title{
A Self-Consistent Model for Positronium Formation from Helium Atoms
}

\author{
E. Ghanbari-Adivi* \\ Department of Physics and Isfahan Quantum Optics Group(IQOG), \\ Faculty of Sciences, University of Isfahan, Isfahan 81746-73441, Iran
}

\begin{abstract}
The differential and total cross sections for electron capture by positrons from helium atoms are calculated using a first-order distorted wave theory satisfying the Coulomb boundary conditions. In this formalism a parametric potential is used to describe the electron screening in a consistent and realistic manner. The present procedure is self consistent because (i) it satisfies the correct boundary conditions and post-prior symmetry, and (ii) the potential and the electron binding energies appearing in the transition amplitude are consistent with the wave functions describing the collision system. The results are compared with the other theories and with the available experimental measurements. At the considered range of collision energies, the results agree reasonably well with recent experiments and theories.
\end{abstract}

PACS numbers: $34.70 .+\mathrm{e}$

Keywords: Distorted-wave approximation. Positronium formation. Coulomb boundary conditions. Differential and total cross sections

\section{INTRODUCTION}

Both positrons $\left(e^{+}\right)$and positronium atoms $(P s)$ have important applications in many different branches of physics, chemistry and other fields [ 1 [ 6$]$. This has motivated numerous studies of collisions in which electrons in atomic or molecular targets are captured by positrons 7 26]. In a collision, each of the various modes into which the system under study may be fragmented is called a channel. For definiteness, consider the positron-helium scattering system, on which the present paper is focused. In the entrance channel of the $e^{+}+\mathrm{He}$ system the positron impacts on the helium atom. The exit channel can be one of a number of possible fragmentations, such as elastic or excitation scattering $\left(e^{+}+H e\right.$ and/or $\left.e^{+}+\mathrm{He}^{*}\right)$, positronium formation $\left(\mathrm{Ps}+\mathrm{He}^{+}\right)$, or ionization $\left(e^{+}+e^{-}+H e^{*}\right)$. An exit channel is said to be open if the corresponding collision is allowed by all known conservation laws, such as energy and momentum conservation; otherwise, it is said to be closed. Since the transition amplitude in general is a function of the energy, the occurrence probability for each open channel depends on the impact energy of the projectile. The transition probabilities per unit time, per unit target scatterer and per unit of the flux of incident particles with respect to the target are called the cross section. Experiments measure cross sections, while theoretical studies usually attempt to compute them. In positron-helium collisions, since the charge transfer process amounts to a four-body problem, the theoretical investigation of the rearrangement involves the full complexities of the quantum mechanical four-body problem and as such is too difficult to be exactly solved.

Notwithstanding the difficulty, numerous theoretical investigations are found in the literature $17-19,33$ 38, 40, 41], along with experimental studies [14, 27 32].

\footnotetext{
* ghanbari@phys.ui.ac.ir
}

Most of the theories were formulated within a single active electron picture. In this formalism, it is important to provide a consistent and realistic treatment of the passive electron screening effects, in order to satisfy the Coulomb boundary conditions and post-prior equivalency and to make the wave functions, binding energies, and Coulomb phase factors consistent with each other.

In the context of K-shell electron capture processes by fast protons from multi electron atoms, Decker and Eichler have discussed the deviations of a few of the standard formalisms from these constraints in some detail [42]. They adopted a parameterized potential due to Green, Sellin and Zocher [43, 44], the GSZ potential, to construct a self-consistent screened boundary-corrected firstBorn-approximation theory for calculation of the K-shell electron capture total cross sections in collisions of proton with helium and carbon as well as collision of alpha particles with lithium atoms.

In the present paper a distorted-wave boundarycorrected first-order Born (DWB1B) formalism accompanied by the GSZ potential is applied to obtain a satisfactory description of positronium formation in positronhelium atom collisions at intermediate energies. In this model, the single-zeta Hartree-Fock wave function and its corresponding binding energy, which show very good agreement with those for GSZ potential, describe the initial bound state of the electron. Consequently the wave function and the corresponding energy in the post or prior formalisms are the eigenfunction and eigenenergy of the same effective screening potential that appears explicitly in the corresponding amplitudes. Thus, in addition to satisfying both the Coulomb boundary conditions an post-prior symmetry, the wave functions, binding energies and Coulomb phase factors are consistent with each other.

The plan of the paper is as follows. Section 2 outlines the formalism. Section 3 presents and discusses the results and compares them with other theories and experimental data. The concluding remarks and a sum- 
mary comprise the last section. Unless otherwise stated, atomic units are used throughout.

\section{THEORY}

Consider a bare ion $P$ with mass $M_{P}$ and charge $Z_{P}$ impingent on an atomic target composed of an active electron $e$ and a residual target ion $T$ with masses $m$ and $M_{T}$, respectively. During the collision, the projectile captures the electron. According to the time-independent boundary-corrected perturbation formalism of electron capture developed by Toshima et al [46] and Belkić et al [47], the first-order corrected-boundary distorted-wave Born(DWB1B) amplitude for such a process has the prior and post forms

$$
\mathcal{A}_{D W B 1 B}^{(\text {prior })}=\left\langle\chi_{f}\left|\left(V_{i}-V_{i}^{\infty}\right)\right| \chi_{i}\right\rangle
$$

and

$$
\mathcal{A}_{D W B 1 B}^{(\text {post })}=\left\langle\chi_{f}\left|\left(V_{f}-V_{f}^{\infty}\right)\right| \chi_{i}\right\rangle,
$$

where $V_{i}^{\infty}$ and $V_{f}^{\infty}$ are the asymptotic limits of the distorting potentials $V_{i}$ and $V_{f}$ in initial and final channels. In position space, the corresponding distorted wavefunctions $\chi_{i}$ and $\chi_{f}$ are given by the equalities

$$
\begin{aligned}
\chi_{i}\left(\mathbf{r}_{T},\right. & \left.\mathbf{R}_{T}, \mathbf{R}\right)=\phi_{i}\left(\mathbf{r}_{T}\right) \exp \left(i \mathbf{K}_{\mathbf{i}} \cdot \mathbf{R}_{T}\right) \\
& \times \exp \left(i \frac{Z_{P}\left(Z_{T}^{a}-1\right)}{\mathrm{v}_{i}} \ln \left(\mathrm{v}_{i} R-\mathbf{v}_{i} \cdot \mathbf{R}\right)\right)
\end{aligned}
$$

and

$$
\begin{aligned}
& \chi_{f}\left(\mathbf{r}_{P}, \mathbf{R}_{P}, \mathbf{R}\right)=\phi_{f}\left(\mathbf{r}_{P}\right) \exp \left(i \mathbf{K}_{\mathbf{f}} \cdot \mathbf{R}_{P}\right) \\
& \quad \times \exp \left(i \frac{Z_{T}^{a}\left(Z_{P}-1\right)}{\mathrm{v}_{f}} \ln \left(\mathrm{v}_{f} R-\mathbf{v}_{f} \cdot \mathbf{R}\right)\right),
\end{aligned}
$$

in which $\phi_{i}\left(\mathbf{r}_{T}\right)$ and $\phi_{f}\left(\mathbf{r}_{P}\right)$ are the initial and final bound-state electronic wave functions. In Eqs. (3) and (4), the plane wave functions describe the heavyparticle motion in the entrance and exit channels, and the phase factors ensure that the overall solutions satisfy the proper asymptotic boundary conditions for the distorting potentials. In these equations, $\mathbf{r}_{P}$ and $\mathbf{r}_{T}$ are the electron coordinates relative to the projectile and target nucleus, respectively, $\mathbf{R}_{P}$ is the position vector of the center of mass of the $P e$ subsystem relative to $T, \mathbf{R}_{T}$ is a similar vector directed from the center of mass of the $T e$ subsystem to $P$, and $\mathbf{R}$ is the internuclear coordinate vector, directed from $T$ to $P$. The bare projectile and the asymptotic target-ion charges are $Z_{P}$ and $Z_{T}^{a}$, respectively, $\mathbf{v}_{i}$ and $\mathbf{v}_{f}$ are the projectile velocity in the initial channel and resultant bound subsystem velocity in the final channel, respectivley, and $\mathbf{K}_{i}$ and $\mathbf{K}_{f}$ are the wave vectors describing the relative motions in the two channels.

The Coulomb interaction between the active electron and the projectile ion is denoted $V_{P e}\left(\mathbf{r}_{P}\right)=-Z_{P} / r_{P}$, with the asymptotic form of $V_{P e}^{\infty}(\mathbf{R})=-Z_{P} / R$. We take the internuclear potential to be that of a bare nucleus screened by the nonactive electrons represented by frozen orbitals. For this purpose, the parameterized potential obtained by Green, Sellin and Zocher (GSZ) [43, 44] is adequate because it has the correct behavior near the nucleus and at infinity and because it yields orbital binding energies and wave functions in good agreement with the corresponding Hartree-Fock results [45]. Within a single active electron model, using the GSZ potential for a neutral atom, one can write the projectile-target interaction in coordinate representation, $V_{P T}(\mathbf{R})$ as

$$
V_{P T}(R)=\frac{Z_{P}}{R}\left[1+\left(Z_{T}-1\right) \Omega(R)\right],
$$

where $Z_{T}$ is the nuclear charge of the multielectron atomic target and $\Omega(R)$ is given by the expression

$$
\Omega(R)=\frac{1}{1-H[1-\exp (R / d)]} .
$$

Optimized values for the characteristic parameters $H$ and $d$ have been given by Szydlik and Green [4] for $Z_{T} \leq 18$. Near the nucleus the projectile ion feels the bare nuclear charge $Z_{T}$, while at large distances the potential behaves as $Z_{P} / R$ corresponding to the residual singly charged ion.

With the potential (5), the post and prior amplitudes (1) and (2) satisfy the appropriate boundary conditions in the initial and final channels. It is easy to see that the two amplitudes are identical provided that $\phi_{i}\left(\mathbf{r}_{T}\right)$ and $\phi_{f}\left(\mathbf{r}_{P}\right)$ be the exact solutions of the atomic Schrödinger equations associated with the potentials $V_{T e}\left(\mathbf{r}_{T}\right)$ and $V_{P e}\left(\mathbf{r}_{P}\right)$, respectively. The wave functions, binding energies and Coulomb phase factors are moreover consistent with each other. Hereafter, given the equivalence between the prior and post wavefunctions, we will omit the superscripts post and prior in Eqs. (11) and (2) and write $\mathcal{A}_{D W B 1 B}$ to denote the amplitudes.

The Fourier transform of the $V_{P T}$ potential can be written in the form

$$
\tilde{V}_{P T}(\mathbf{k})=\sqrt{\frac{2}{\pi}} \frac{Z_{P}}{k^{2}}+\sqrt{\frac{2}{\pi}} Z_{P}\left(Z_{T}-1\right) \tilde{\mathcal{V}}(k),
$$

where

$$
\tilde{\mathcal{V}}(k)=\frac{1}{k} \int_{0}^{\infty} d R \Omega(R) \sin (k R) .
$$

With the given interaction potentials, $V_{P e}\left(\mathbf{r}_{P}\right)$ and $V_{P T}(\mathbf{R})$, the transition amplitude for electron capture comprises three terms:

$$
\mathcal{A}_{D W B 1 B}=\mathcal{A}_{1}+\mathcal{A}_{2}+\mathcal{A}_{3},
$$

with the following explicit expressions for the partial am- 
plitudes $\mathcal{A}_{1}, \mathcal{A}_{2}$ and $\mathcal{A}_{3}$ in coordinate representation:

$$
\begin{gathered}
\mathcal{A}_{1}=-Z_{P} \int d \mathbf{r}_{P} d \mathbf{R}_{P} \chi_{f}^{*}\left(\mathbf{r}_{P}, \mathbf{R}_{P}, \mathbf{R}\right) \frac{1}{r_{P}} \chi_{i}\left(\mathbf{r}_{T}, \mathbf{R}_{T}, \mathbf{R}\right) \\
\mathcal{A}_{2}=+Z_{P} \int d \mathbf{r}_{P} \mathbf{R}_{P} \chi_{f}^{*}\left(\mathbf{r}_{P}, \mathbf{R}_{P}, \mathbf{R}\right) \frac{1}{R} \chi_{i}\left(\mathbf{r}_{T}, \mathbf{R}_{T}, \mathbf{R}\right) \\
\mathcal{A}_{3}=Z_{P}\left(Z_{T}-1\right) \int d \mathbf{r}_{P} d \mathbf{R}_{P} \chi_{f}^{*}\left(\mathbf{r}_{P}, \mathbf{R}_{P}, \mathbf{R}\right) \frac{\Omega(R)}{R} \\
\times \chi_{i}\left(\mathbf{r}_{T}, \mathbf{R}_{T}, \mathbf{R}\right) .
\end{gathered}
$$

We now consider the special case $Z_{P}=1$ and the transition from an initial $1 s$ hydrogen-like state such as $\phi_{i}\left(\mathbf{x}_{T}\right)=N_{T} \exp \left(-\zeta_{T} x_{T}\right)$ to a final $1 s$ hydrogen-like state of form $\phi_{f}\left(\mathbf{x}_{P}\right)=N_{P} \exp \left(-\zeta_{P} x_{P}\right)$, where $N_{P}$ and $N_{T}$ are normalization factors. We apply the Scrödinger equation for the final bound subsystem, use the Fourier transform analysis, and evaluate the resulting integrals to obtain the following expression for the first partial amplitude, $\mathcal{A}_{1}$ :

$$
\begin{aligned}
\mathcal{A}_{1}=-16 \pi^{2} N_{P} N_{T} Z_{P}\left(\frac{K^{2}}{2 \mu_{f}}-\epsilon_{f}\right) \\
\times \frac{\partial^{2}}{\partial \zeta_{P} \partial \zeta_{T}}\left[\frac{1}{\left(K^{2}+\zeta_{P}^{2}\right)\left(J^{2}+\zeta_{T}^{2}\right)}\right],
\end{aligned}
$$

where $\mu_{f}=M_{P} m /\left(M_{P}+m\right)$ is the reduced final-state mass of the bound subsystem, and $\mathbf{J}$ and $\mathbf{K}$ are the momenta transferred to the target ion and the projectile, respectively, during the collision.

Similarly, using the Fourier transform techniques and Lewis integral [48], we derive the following closed form for the second partial amplitude $\mathcal{A}_{2}$ :

$$
\begin{aligned}
& \mathcal{A}_{2}=8 \pi^{2} N_{P} N_{T} Z_{P} \\
& \times \frac{\partial^{2}}{\partial \zeta_{P} \partial \zeta_{T}}\left[\left(\alpha^{2}-\beta\right)^{-1 / 2} \ln \left[\frac{\alpha+\left(\alpha^{2}-\beta\right)^{1 / 2}}{\alpha-\left(\alpha^{2}-\beta\right)^{1 / 2}}\right]\right],
\end{aligned}
$$

with

$$
\alpha=\left(K^{2}+\zeta_{P}^{2}\right) \zeta_{T}+\left(J^{2}+\zeta_{T}^{2}\right) \zeta_{P}
$$

and

$$
\beta=\left(K^{2}+\zeta_{P}^{2}\right)\left(J^{2}+\zeta_{T}\right)\left[v_{f}^{2}+\left(\zeta_{P}^{2}+\zeta_{T}^{2}\right)\right] .
$$

Finally, we use Fourier analysis to write the third partial amplitude $\mathcal{A}_{3}$ in the form

$$
\mathcal{A}_{3}=8 \pi^{2} N_{P} N_{T} Z_{P}\left(Z_{T}-1\right) \frac{\partial^{2}}{\partial \zeta_{P} \partial \zeta_{T}} \mathcal{I}\left(\zeta_{P}, \zeta_{T}\right),
$$

where

$$
\mathcal{I}\left(\zeta_{P}, \zeta_{T}\right)=\frac{1}{\pi^{2}} \int d \mathbf{k} \frac{\tilde{\mathcal{V}}(k)}{\mid\left(\mathbf{k}-\left.\mathbf{K}\right|^{2}+\zeta_{P}^{2}\right)\left(\mathbf{k}+\left.\mathbf{J}\right|^{2}+\zeta_{T}^{2}\right)} .
$$

Before numerical calculations become possible, we have to analytically simplify the right-hand side of Eq. (13).
To this end, notice taken that $\tilde{\mathcal{V}}(k)$ is a radial function, we use Feynman's identity [49] to evaluate the angular integral over $\mathbf{k}$ :

$\mathcal{I}\left(\zeta_{P}, \zeta_{T}\right)=-\frac{1}{\pi} \int_{0}^{1} \frac{d x}{\gamma} \int_{0}^{\infty} d R \Omega(R) \int_{-\infty}^{+\infty} d k \frac{\sin (k R)}{k^{2}+2 \gamma k+\delta}$,

where

$$
\gamma=\sqrt{J^{2}(1-x)^{2}+K^{2} x^{2}-2 x(1-x) \mathbf{J} \cdot \mathbf{K}},
$$

and

$$
\delta=\left(J^{2}+\zeta_{T}^{2}\right)(1-x)+\left(K^{2}+\zeta_{P}^{2}\right) x .
$$

Next, we apply Cauchy's residue theorem to the last integral on the right-hand side of Eq. (15) to see that

$$
\int_{-\infty}^{+\infty} d k \frac{\sin (k R)}{k^{2}+2 \gamma k+\delta}=-\frac{\pi}{\sqrt{\delta-\gamma^{2}}} \mathrm{e}^{-R \sqrt{\delta-\gamma^{2}}} \sin (\gamma R) .
$$

With this, we have reduced the four-dimensional integral $\mathcal{I}\left(\zeta_{P}, \zeta_{T}\right)$ in Eq. (14) to a two dimensional form,

$\mathcal{I}\left(\zeta_{P}, \zeta_{T}\right)=\int_{0}^{1} \frac{d x}{\gamma \sqrt{\delta-\gamma^{2}}} \int_{0}^{\infty} d R \Omega(R) \mathrm{e}^{-R \sqrt{\delta-\gamma^{2}}} \sin (\gamma R)$,

which can be easily computed numerically, along with the derivatives on the right-hand side of Eq. (13).

For single-electron capture in impact of positron on helium atoms $Z_{T}=2.0, \epsilon_{i}=-0.89648, \epsilon_{f}=-0.25$, $\mu_{f}=0.5, \zeta_{P}=0.5, \zeta_{T}=1.6875, N_{P}=\zeta_{P} \sqrt{\zeta_{P} / \pi}$, $N_{T}=\zeta_{T} \sqrt{\zeta_{T} / \pi}$ and the corresponding differential and total cross sections are given by

$$
\sigma(\theta)=\frac{d \sigma}{d \Omega}=\frac{2}{\pi^{2}} \frac{v_{f}}{v i}\left|\mathcal{A}_{D W B 1 B}\right|^{2}
$$

and

$$
\sigma_{\text {Total }}=2 \pi \int_{0}^{\pi} \sigma(\theta) \sin \theta d \theta
$$

respectively.

To account for the excited final states, we multiply the right-hand side of Eq. (19) by 1.202, according to the Oppenheimer $n^{-3}$ scaling rule [50].

\section{RESULTS AND CONCLUSIONS}

We now present the computed differential and total cross sections for positronium formation in positron collisions with helium atoms and compare them with other theoretical approaches and experimental data. In order to evaluate the third partial transition amplitude, $\mathcal{A}_{3}$, we rely on the Gaussian quadrature method to compute the two-dimensional integral $\mathcal{I}\left(\zeta_{P}, \zeta_{T}\right)$ in Eq. (17).

Figure 1 shows the angular differential cross sections for positronium formation at three incident energies, 100, 

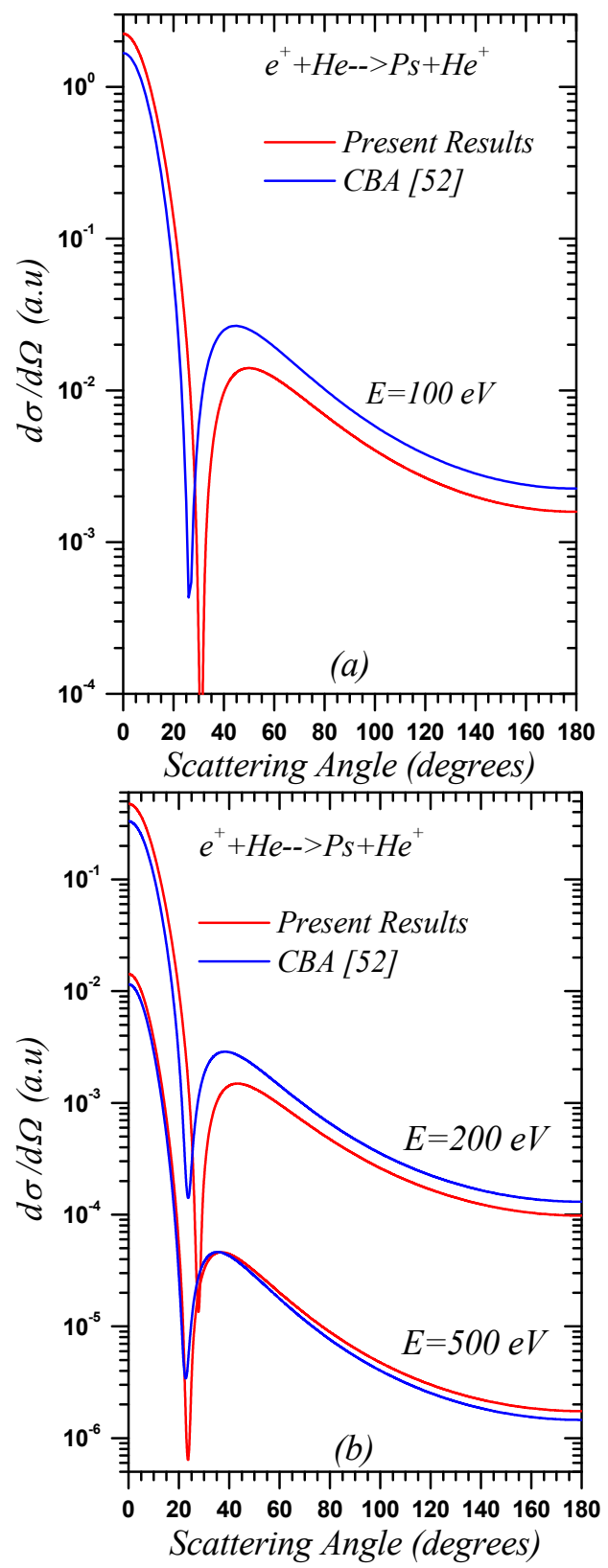

FIG. 1. Angular distribution of the differential cross sections for Ps formation from helium atoms.

200 and $500 \mathrm{eV}$. All three curves display a dip due to the cancelation between the contributions of the attractive and repulsive interactions to the first-order transition amplitude. A similar, unphysical dip has been found in a first-order Born treatment of electron capture in the collision of a proton with hydrogen atoms [51]. Figures 1(a) and (b) compare our results with those of the CoulombBorn approximation (CBA) 52 for three impact energies: 100, 200 and $500 \mathrm{eV}$. In our computation, the dark angles, i. e., the angles at which cancelation occurs, are $31^{\circ}, 28^{\circ}$ and $24^{\circ}$, while the CBA displays dark angles of $26^{\circ}, 24^{\circ}$ and $23^{\circ}$, respectively. In both treatments, the dark angle becomes smaller as the incident energy grows.
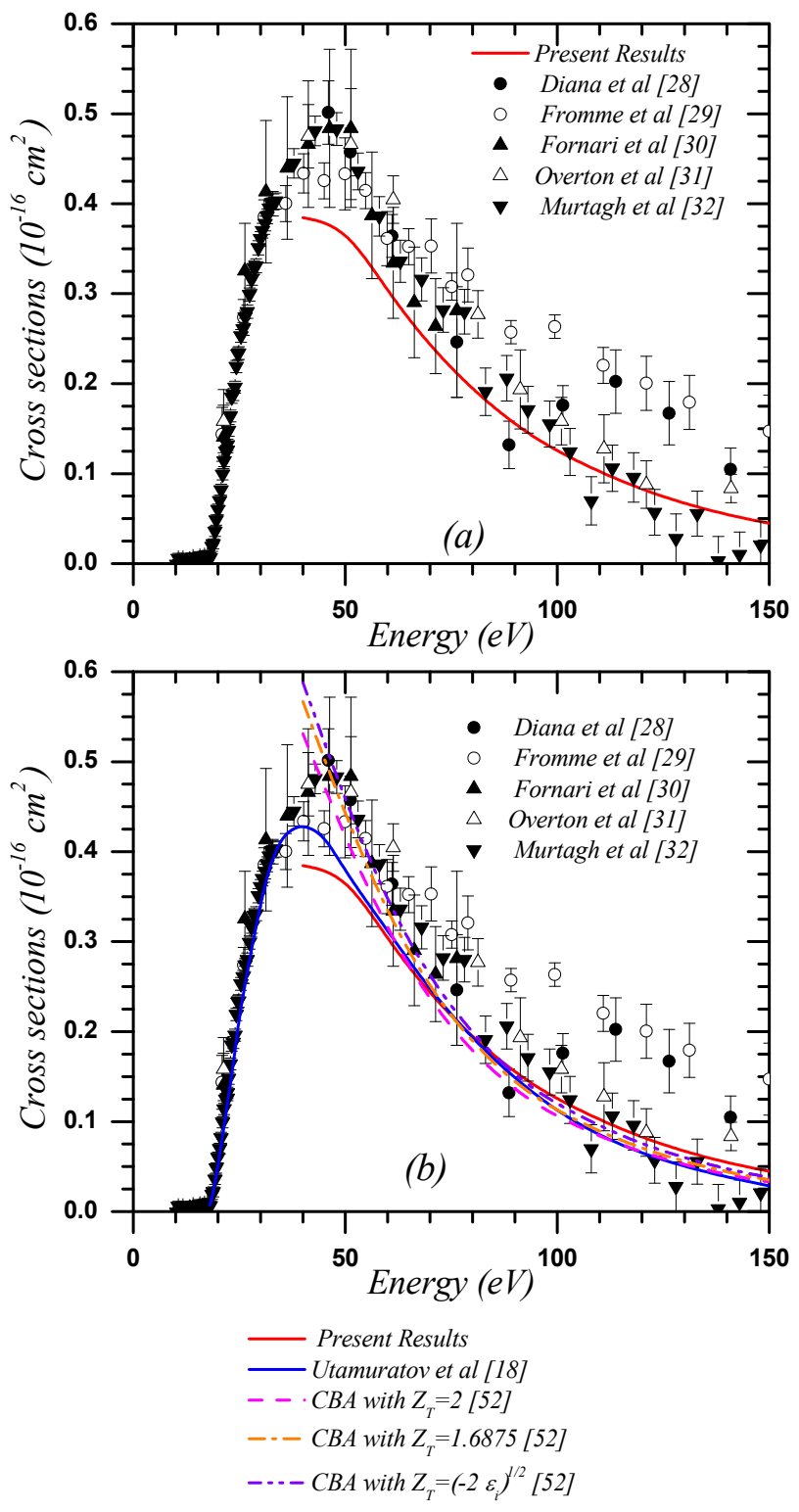

FIG. 2. Total cross sections for Ps formation in collisions of positrons with helium atoms, compared with the results of the listed theoretical and experimental studies.

Our results differ significantly from the CBA data at $100 \mathrm{eV}$ incident energy. Below (above) $30^{\circ}$ scattering angle our differential cross sections are always smaller (larger) than the CBA results. As the impact energy increases, the difference between the our results and the CBA results shrinks. Compared with the difference at $100 \mathrm{eV}$, the difference at $200 \mathrm{eV}$ is smaller, and the difference at $500 \mathrm{eV}$ is negligible. For energies above $500 \mathrm{eV}$, our results are consistent with the CBA results. Consequently, the total cross sections obtained from the two formalisms converge at high energies. Figure 2 compares the total positronium-formation cross sections obtained from our formalism with the experimental measurements of Diana et al 28], Fromme et al [29], Fornari et al [30], 
Overton et al 31] and Murtagh et al 32] and also with the results of the $C B A$ with $Z_{T}=1.6875,2$ and $\sqrt{-2 \epsilon_{i}}$ [52] and of the two-center convergent close-coupling (CCC) formalism [18]. As Fig. 2(a) shows, in spite of its simplicity our formalism yields reasonable agreement with experiment at energies above $50 \mathrm{eV}$. Both the present approach and the CBA formalism [52] are based on perturbation expansion, an approach long known to be unreliable at low scattering energies.

For the first-order Born approximation to be accurate, the higher-order terms in the expansion series must be small in comparison with the first-order term. At high energies, since two-step scattering processes, such as the Thomas double-scattering mechanism, become dominant at high energies, the first-order approach yields poor approximations to the differential cross sections. All considered, we see that first-order methods are only reliable in a bracket of energies not so low as to render the perturbative method inapplicable and not so high as to make double-scattering mechanisms dominant.

Both the CBA and our approach satisfy the correct boundary conditions. The two methods are nonetheless nonequivalent: while the CBA breaks post-prior symmetry, our approach is symmetric, so that the initial and final wavefunctions associated with GSZ potential are consistent with the scattering potential and bound energies. Our approach is hence expected to be more appropriate to describe experimental processes. As the plots in Fig. 2(b) show, however, only small differences separate the CBA results from ours.

More accurate positronium-formation cross sections are obtained with the CCC formalism, a more elaborate method employing a multiconfigurational wavefunction treatment to describe the He ground state. The accuracy of the calculated cross sections is controlled by the size of the basis and of the set quantum numbers of the included states at each center. Figure 2(b) shows good agreement between the CCC curve and the experimental data.

Within the energy bracket in which it is reliable, the solid line depicting our results in Fig. 2(b) agrees with the $\mathrm{CCC}$ line. The difference between the two sets theoretical data is less than $0.7 \%$ around $50 \mathrm{eV}$, while deviations of approximately $17 \%$ separate the CBA and CCC curves in the same region. At higher energies the results of the three formalisms converge and display good agreement with the experimental data.

Figure 3 compares various theoretical approaches with experiment. Cross sections calculated by the CBA with $Z_{T}=1.6875,2$ and $\sqrt{-2 \epsilon_{i}}[52]$, the correct-boundary first-order Born (B1B) 38] formalism, the distorted-wave Born (DW) approximation [39], the continuum distorted wave (CDW) approximation [40] and the close-coupling method (CC) [41] are compared with the experiments of Fornari et al [30] and Overton et al [31]. The CBA, $\mathrm{CDW}$, and $\mathrm{CC}$ agree well with the measurments in the depicted energy range. The agreement between B1B and DW at high energies is fair, but at lower energies those

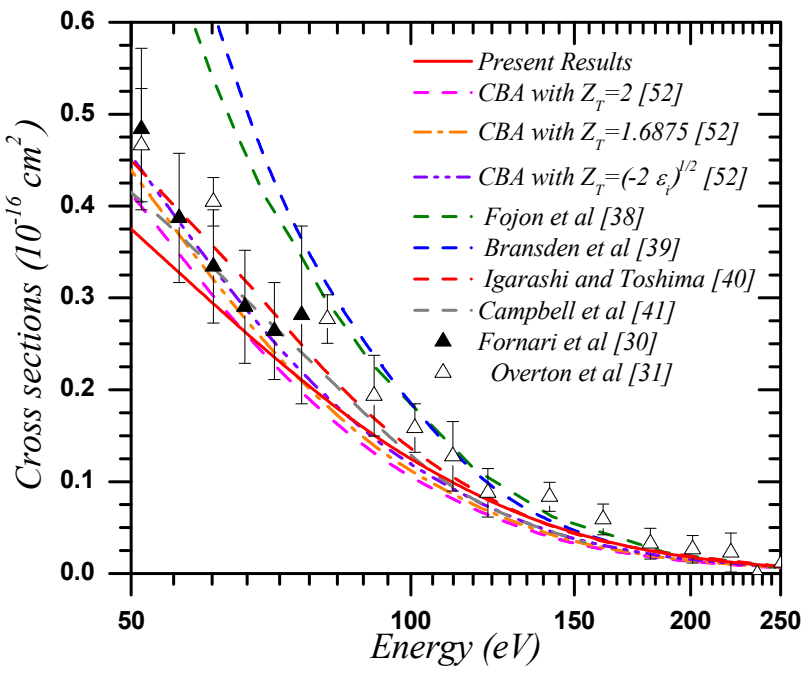

FIG. 3. Total cross sections, compared with the results of the CBA [52], B1B [38], DW 39], CDW [40] and CC [41] procedures and with the experimental data from Fornari et al 30] and Overton et al [31].

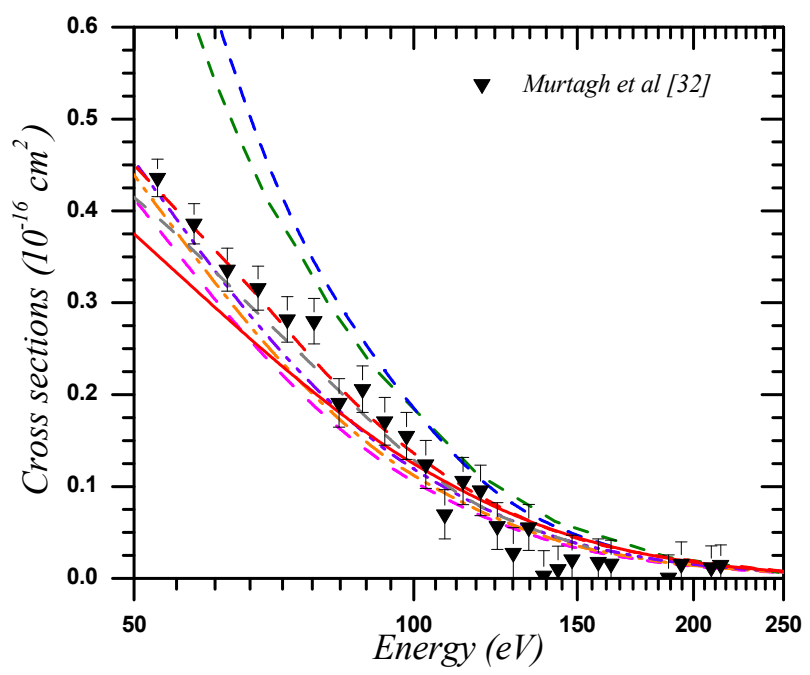

FIG. 4. Theoretical cross sections, as in Fig. 3, compared with the measurements by Murtagh et al 32]

theories deviate from the measurements.

Our predictions also deviate from experiment at low energies, but the agreement improves as the energy increases. Compared with the results of B1B and DW, our results are closer to the experimental data at low energies.

Figure 4 compares the above discussed theories with the earlier experimental measurements reported by Murtagh et al [32]. The CDW, CC, and CBA formalisms yield results in very good agreement with experiment over the entire energy range. Our results are also in good agreement at energies above $75 \mathrm{eV}$, but the discrepancies are sizable at lower energies. The B1B and DW results are above the data by Murtagh et al [32] for all energies 


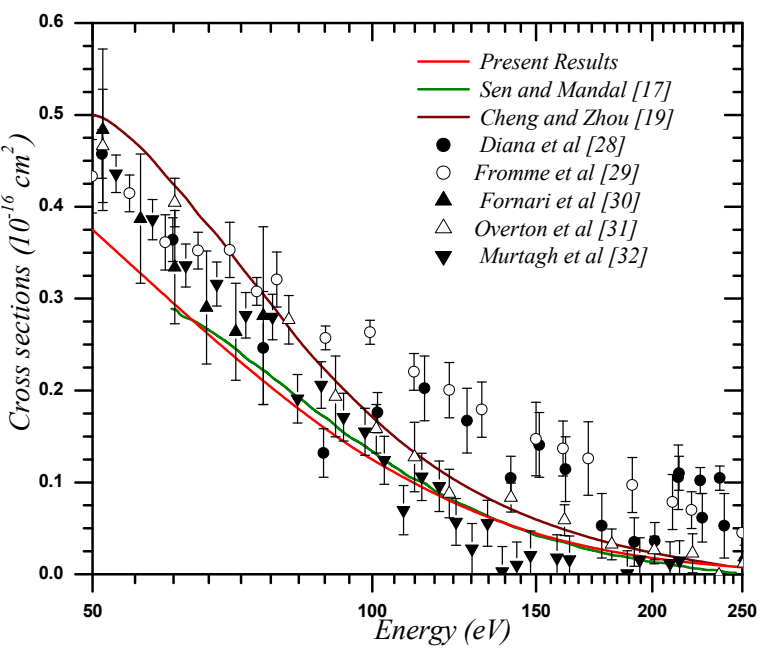

FIG. 5. Cross sections for positronium formation in helium atoms compared with all available experimental data and with the results of the DWA [17] and CCO [19] formalisms.

lower than $200 \mathrm{eV}$, the deviation growing considerably as the impact energy decreases. Above $200 \mathrm{eV}$, all theories converge to the experimental data.

In addition to the CCC formalism, a number of theoretical descriptions of positronium formation in positronhelium atom collisions have been recently reportde, on the basis of the momentum-space coupled-channel optical (CCO) method [19] and second-order distorted-wave approximation (DWA) [17]. Figure 5 compares our results in the energy range of $50-250 \mathrm{eV}$ with the available results from the CCO and DWA formalisms, as well as with the experimental data reported by different groups [28 32]. Our first-order distorted wave results are very close to the predictions of the second-order DWA, in which the cross sections for Ps formation in the 1s orbital has been added to 1.66 times the Ps cross sections in the $n=2$ orbitals to yield the total Ps formation cross sections.

Below $80 \mathrm{eV}$ our results understimate the experimental cross sections. At small energies all theories in the plot become inaccurate, the the CCO cross sections overshooting the data by Murtagh et al 32]. At relatively higher energies, above $80 \mathrm{eV}$ our results agree with the other theoretical predictions and with the measurements.

Figure 6] shows the total positronium-formation cross sections in our calculation formalism and all the abovementioned theoretical calculations. Comparison with measurements reported by Murtagh et al [32] identifies the CDW [40], CC [41] and CBA (with $Z=2$ ) as the most reliable ones. As before, our data show only fair agreement with these three theories, at low energies, and good agreement at higher energies. Compared with the results of Fojón et al [8] and of Bransden et al [39], our results show substantially superior agreement with experiment low energies. In the considered range of impact energies, our results are compatible with those from the more complex CCC formalism.
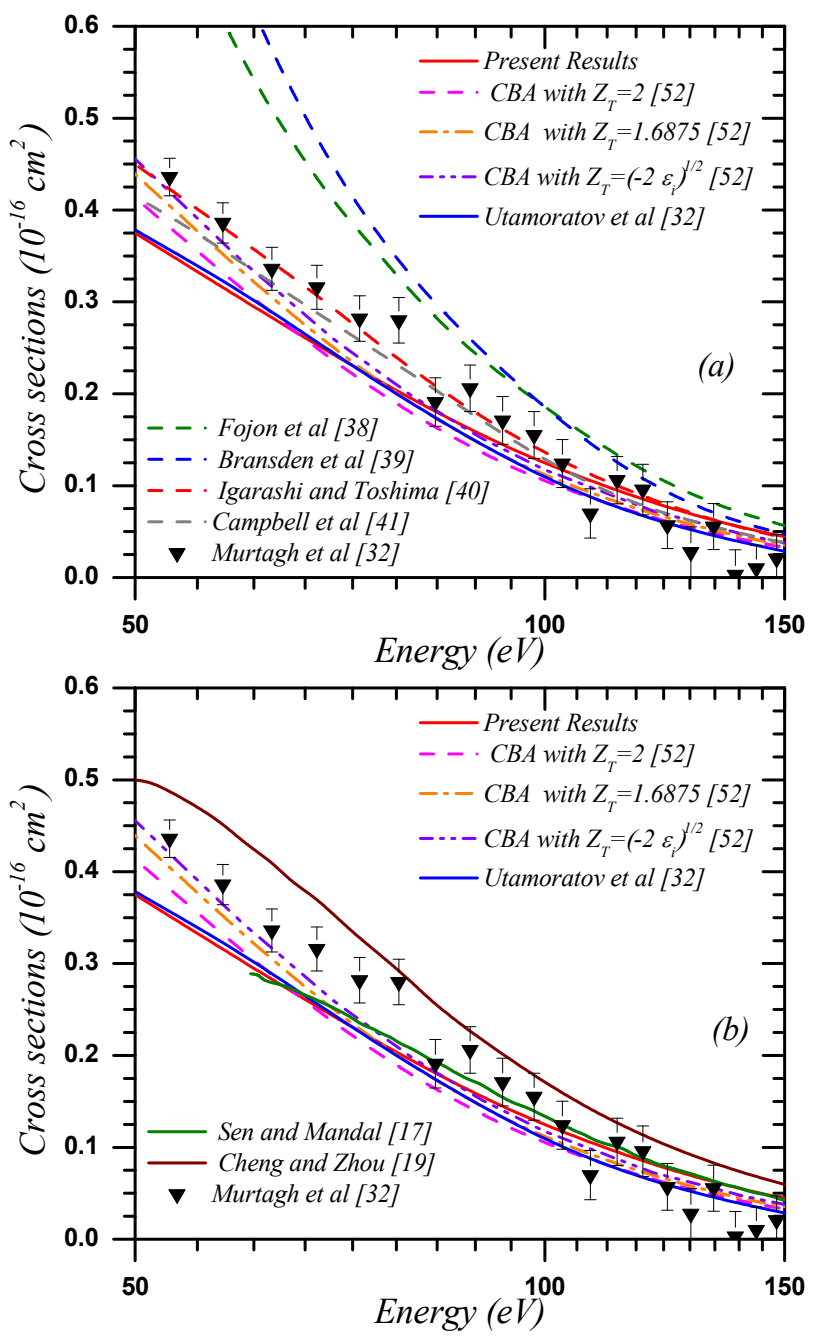

FIG. 6. Total cross sections for Ps formation from helium atoms compared the results of the listed theories and with the experimental data by Murtagh et al 32].

\section{SUMMARY AND CONCLUSIONS}

In summary, we have applied a first-order distorted wave with correct boundary conditions to positronium formation from helium atoms. A parameterized potential in good agreement with Roothaan-Hartree-Fock wavefunction and the corresponding binding energy described the screening effect of the passive electron on the transition amplitude. Our theory is self-consistent, since it satisfies the correct boundary conditions and the post-prior symmetry, and since the interaction potentials, wavefunctions, binding energies and Coulomb phase factors in the formalism are consistent with each other and describe the collision and electron capture realistically.

The chief advantage of our approach is its simplicity. The figures in this paper comprehensively compare the results of this approach with different sets of experimental data and with the results of more elaborate theories. They hence set benchmarks monitoring the accuracy of 
the treatment. Compared with the results of the CBA, the angular distribution of our differential cross sections are in qualitative agreement even at the smallest energies: they differ quantitatively at the smallest energies, but the agreement improves as the impact energy grows.

Our calculated total cross sections are in reasonable agreement with other theories, such as the CBA, CDW, CC, CCO, and DWA, especially at higher energies. Also the results agree well with with the experimental data of Fornari et al [30], Overton et al [31], and Murtagh et al [32], the agreement with the latter authors being very good at incident energies above $75 \mathrm{eV}$. As evidenced by comparison with experimental and other theoretical data, our procedure becomes less accurate as the impact energy is reduced, significant deviations becoming apparent around $50 \mathrm{eV}$.

Our relatively simple approach, based on the first- order distorted-wave formalism reproduces the results of more demanding procedures, based on the secondorder distorted wave approximation. It can be applied to positronium formation in multi-electron atoms, such a $\mathrm{C}, \mathrm{Ne}, \mathrm{Na}$, and $\mathrm{Ar}$, and also to electron caputre from atomic shells of multi-electron atoms by bare ion projectiles at moderate energies.

\section{ACKNOWLEDGMENTS}

The author would like to thank Dr. Daniel Murtagh for providing the experimental data which were measured by his group (UCL positron group) on the different positron scattering processes from helium atoms and Dr. Ravshan Utamoratov for forwarding his results for Ps formation based on the two-center convergent close-coupling formalism in tabular form.
[1] Charlton M and Humberston J W 2001 Positron Physics (Cambridge: Cambridge University Press)

[2] Surko C M and Gianturco F A 2001 New Directions in Antimatter Chemistry and Physics (Kluwer Academic Publishers, Dordrecht)

[3] Jean Y C, Mellon P E, Schrader D M 2003 Principles and Applications of Positron and Positronium Chemistry (World Scientific Publishing Company, Singapore)

[4] Puska J M and Nieminen R M 1994 Rev. Mod. Phys. 66 841

[5] David A, Kögel G, Sperr P and Triftshäuser W 2001 Phys. Rev. Lett. 87, 067402

[6] Jerusalem G, Hustinx R, Beguin Y and Fillet G 2003 Eur. J. Cancer 391525

[7] Gribakin G F and Ludlow J 2004 Phys. Rev. A 70032720

[8] Fojón O Hanssen J Hervieux P A and Rivarola R D 2000 J. Phys. B: At. Mol. Opt. Phys. 333093

[9] Laricchia G, Brawley S, Cooke, Kövér Á, Murtagh D J and Williams A I 2009 J. Phys. : Conference Series 194 012036

[10] Cooke D A, Murtagh D J and Laricchia G 2010 J.Phys.: Conference Series 199012006

[11] Dunlop L J M and Gribakin G F 2006 Nucl. Inst. Meth. Phys. Res. B 24761

[12] Macri P A and Barrachina R O 2008 Nucl. Inst. Meth. Phys. Research B 266393

[13] Macri P A and Barrachina R O 2009 Nucl. Instr. Meth. B267366

[14] Caradonna P, Sullivan J P, Jones A, Makochekanwa C and Buckman S J 2009 Phys. Rev. A 80060701

[15] Surdutovich E, Johnson J M, Kauppila W E, Kwan C K and Stein T S 2002 Phys. Rev. A 65032713

[16] Surdutovich E, Harte M, Kauppila W E, Kwan C K and Stein T S 2003 Phys. Rev. A 67022709

[17] Sen S and Mandal P 2009 Phys. Rev. A 80062714

[18] Utamuratov R, Kadyrov A S, Fursa D V, Bray I and Stelbovics A T 2010 J. Phys. B: At. Mol. Opt. Phys. 43 125203

[19] Cheng Y and Zhou Y 2007 Phys. Rev. A 76012704
[20] Cheng Y and Zhou Y 2006 Phys. Rev. A 73024701

[21] Marler J P, Sullivan J P and Surko C M 2005 Phys Rev A $\mathbf{7 1} 022701$

[22] Le A T, Bromley M W J and Lin C D 2005 Phys Rev A 71032713

[23] Nan G, Zhou Y and Ke Y 2005 Phys. Rev. A 72012709

[24] Murtagh D J, Cooke D A and Laricchia G 2009 Phys. Rev. Lett 102133202

[25] Ke Y, Zhou Y and Nan G 2004 Phys. Rev. A 70024702

[26] Kauppila W E, Miller E G, Mohamed H F M, Pipinos K, Stein T S and Surdutovich E 2004 Phys. Rev. Lett. 93, 113401

[27] Charlton M, Clark G, Griffith T C and Heyland G R 1983 J. Phys. B: At. Mol. Phys. 16 L465

[28] Diana L M, Coleman P G, Brooks D L, Pendleton P K and Norman D M 1986 Phys. Rev. A 34, 2731

[29] Fromme D, Kruse G, Raith W and Sinapius G 1986 Phys. Rev. Lett. 57, 3031

[30] Fornari L S, Diana L M and Coleman P G 1983 Phys. Rev. Lett. 51, 2276

[31] Overton N, Mills R J, Coleman P G 1993 J. Phys. B: At. Mol. Opt. Phys. 26, 3951

[32] Murtagh D J, Szłuińska M, Moxom J, Van Reeth P and Laricchia G 2005 J. Phys. B: At. Mol. Opt. Phys. 383857

[33] Hewitt R N, Noble C J and Bransden B H 1992 J. Phys. B: At. Mol. Opt. Phys. 25557

[34] Sarkar N K, Basu M and Ghosh A S 1992 Phys. Rev. A 456887

[35] Schultz D R and Olson R E 1998 Phys. Rev. A 381866

[36] Mandal P, Guha S and Sil N C 1979 J. Phys. B: At. Mol. Phys. 122913

[37] Van Reeth P and Humberston J W 1999 J. Phys. B: At. Mol. Opt. Phys. 323651

[38] Fojón O A, Gayet R, Hanssen J and Rivarola R D 1995 Phys. Scr. 51204

[39] Bransden B H, Joachain C H and McCann J F 1992 J. Phys. B : At. Mol. Opt. Phys. 254965

[40] Igarashi A and Toshima N 1992 Phys. Lett. A 16470 
[41] Campbell C P, McAlinden M T, Kernoghan A A and Walters H R J 1998 Nucl. Inst. Meth. B 14341

[42] Decker F and Eichler J 1989 Phys. Rev. A 391530

[43] Green A E S, Sellin D L and Zachor A S 1969 Phys. Rev. 1841

[44] Szydlik P P and Green A E S 1974 Phys Rev A 91885

[45] Clementi E and Roetti C 1974 At. Data and Nucl. Data Tab. 14177
[46] Toshima N, Ishihara T and Eichler J 1987 Phys. Rev. A 362659

[47] Belkić Dž, Saini S and Taylor H S 1986 Z. Phys. D 359

[48] Lewis R R Jr 1956 Phys. Rev. 102537

[49] Feynman R P 1949 Phys. Rev. 76769

[50] Oppenheimer J R 1928 Phys. Rev. A 31349

[51] Band Y B 1973 Phys. Rev. A 8243

[52] Ghanbari-Adivi E 2011 Eur. Phys. J. D 62389 